Pacific Journal of Mathematic 


\title{
CONTINUOUS SELECTIONS AND FINITE-DIMENSIONAL SETS
}

\author{
E. MiCHAEL
}

\begin{abstract}
Some known selection theorems are strengthened by weakening the hypotheses on a finite-dimensional subset of the domain.
\end{abstract}

1. Introduction. The following selection theorem was recently obtained in [7] by C. Pixley and the author.

THEOREM 1.1. Let $X$ be paracompact, $Y$ a Banach space, $Z \subset X$ with $\operatorname{dim}_{X} Z \leqq 0$, and $\phi: X \rightarrow \mathscr{F}(Y)$ l.s.c. with $\phi(x)$ convex for all $x \in X-Z$. Then $\phi$ has a selection.

Let us quickly define our terms: We write $2^{Y}=\{S \subset Y: S \neq \varnothing\}$ and $\mathscr{F}(Y)=\left\{S \in 2^{Y}: S\right.$ closed in $\left.Y\right\}$. A map $\phi: X \rightarrow 2^{Y}$ is lower semicontinuous, or l.s.c., if $\{x \in X: \phi(x) \cap V \neq \varnothing\}$ is open in $X$ for every open $V$ in $Y$. A selection for a map $\phi: X \rightarrow 2^{Y}$ is a continuous $f: X \rightarrow Y$ such that $f(x) \in \phi(x)$ for every $x \in X$. Finally, if $Z \subset X$, then $\operatorname{dim}_{X} Z \leqq n$ means that $\operatorname{dim} S \leqq n$ for every subset $S$ of $Z$ which is closed in $X .^{1}$

The purpose of this paper is first to generalize Theorem 1.1 to the case where $\operatorname{dim}_{X} Z \leqq n$, and then to prove two other theorems of this type in which different assumptions are made on $\phi(x)$ when $x \in X-Z$. Just like Theorem 1.1, all of these "hybrid" selection theorems reduce to known results when $Z=\varnothing$ or $Z=X$. Theorem 1.1, along with the special cases of Theorems 1.3 and 1.4 where $\operatorname{dim} Z=0$, will be applied in [6].

In contrast to Theorem 1.1, the theorems to be proved in this paper all have both a local and a global version. To state these results succinctly, we introduce some more terminology: A map $\phi: X \rightarrow 2^{Y}$ has the selection extension property, or SEP, if, whenever $A \subset X$ is closed, every selection $g$ for $\phi \mid A$ extends to a selection for $\phi$. If $g$ only extends to a selection for $\phi \mid U$ for some neighborhood $U$ of $A$ in $X$, then we say that $\phi$ has the selection neighborhood extension property, or SNEP. As pointed out in [6], Theorem 1.1 can be strengthened to conclude that $\phi$ actually has the SEP.

Before stating Theorem 1.2, we recall the following concepts from [4, p. 565], where $n \geqq-1$ : A space $S$ is $C^{n}$ if every continuous image of an $i$-sphere $(i \leqq n)$ in $S$ is contractible in $S$. A collec-

\footnotetext{
${ }^{1}$ Observe that, for normal $X$, $\operatorname{dim}_{X} Z \leqq n$ is valid if either $\operatorname{dim} Z \leqq n$ or $\operatorname{dim} X \leqq n$.
} 
tion $\mathscr{S}$ of subsets of a metric space $Y$ is uniformly equi-LC ${ }^{n}$ if to every $\varepsilon>0$ corresponds a $\nu(\varepsilon)>0$ such that, for every $S \in \mathscr{S}$, every continuous image of an $i$-sphere $(i \leqq n)$ in $S$ of diameter $<\nu(\varepsilon)$ is contractible over a subset of $S$ of diameter $<\varepsilon$. Since there is no $(-1)$-sphere, the above properties are always vacuously satisfied when $n=-1$, and hence Theorem 1.2 really generalizes Theorem 1.1 .

THEOREM 1.2. Let $X$ be paracompact, $Y$ a Banach space, $Z \subset X$ with $\operatorname{dim}_{X} Z \leqq n+1$, and $\phi: X \rightarrow \mathscr{F}(Y)$ l.s.c. with $\phi(x)$ convex for all $x \in X-Z$ and with $\{\phi(x): x \in Z\}$ uniformly equi-LC $C^{n}$. Then $\phi$ has the SNEP. If, moreover, $\phi(x)$ is $C^{n}$ for every $x \in Z$, then $\phi$ has the SEP.

Theorem 1.2 reduces to [3, Theorem $\left.3.2^{\prime \prime}\right]$ when $Z=\varnothing$, and it implies [4, Theorem 1.2] when $Z=X$ (more generally, when $Z$ is open in $X$ and $\phi(x)$ is a singleton for $x \in X-Z)$.

In our next theorem, a metric space $Y$ is called an AR (resp. ANR) if it is a retract (resp. neighborhood retract) of every metric space $E$ containing it as a closed subset. A collection $\mathscr{S}$ of subsets of a topological space $Y$ is called equi-LC in $Y$ if, for every $y \in Y$, every neighborhood $V$ of $y$ contains a neighborhood $W$ of $y$ such that, for any $S \in \mathscr{S}$, every continuous image of an $i$-sphere $(i \leqq n)$ in $W \cap S$ is contractible in $V \cap S$. It is easy to see that every uniformly equi- $L C^{n}$ collection of subsets of a metric space $Y$ is equi- $L C^{n}$ in $Y$; for a partial converse, see Lemma 6.1(b).

THEOREM 1.3. Let $X$ be paracompact, $Y$ a complete metric ANR, $Z \subset X$ with $\operatorname{dim}_{X} Z \leqq n+1$, and $\phi: X \rightarrow \mathscr{F}(Y)$ l.s.c. with $\phi(x)=Y$ for $x \in X-Z$ and with $\{\phi(x): x \in Z\}$ equi-LC $C^{n}$ in $Y$. Then $\phi$ has the SNEP. If moreover, $Y$ is an $\mathrm{AR}$ and $\phi(x)$ is $C^{n}$ for every $x \in Z$, then $\phi$ has the SEP.

Observe that, when $Z=\varnothing$, Theorem 1.3 reduces to the known result that every complete metric AR (resp. ANR) has the extension property (resp. neighborhood extension property) with respect to paracompact spaces (see, for instance, C. H. Dowker [1]).

Our next result reduces to [4, Theorem 1.2] when $Z=\varnothing$.

THeOREM 1.4. Let $X$ be paracompact, $A \subset X$ closed with $\operatorname{dim}_{X}(X-A) \leqq n+1, \quad Z \subset X-A$ with $\operatorname{dim}_{X} Z \leqq m+1$ (where $m \leqq n), \quad Y$ complete metric, and $\phi: X \rightarrow \mathscr{F}(Y)$ l.s.c. such that $\{\phi(x): x \in X-Z\}$ is equi- $L C^{n}$ in $Y$ and $\{\phi(x): x \in Z\}$ is equi- $L C^{m}$ in $Y$. 
Then $\phi$ has the SNEP at $A .^{2}$ If, moreover, $\phi(x)$ is $C^{n}$ for all $x \in X-Z$ and $C^{m}$ for all $x \in Z$, then $\phi$ has the SEP at $A$.

The paper is arranged as follows. Two known lemmas on l.s.c. maps are stated in $\$ 2$, some general results on selections are established in $\S 3$, and a result which is implicitly contained in [4] is recorded in $\S 4$. After these preliminaries, Theorems 1.2, 1.3 and 1.4 are then proved in $\S \S 5,6$ and 7 , respectively.

2. Two lemmas on 1.s.c. maps. The following two known lemmas will be applied in the sequel.

Lemma 2.1 [3, Example 1.3]. Let $\phi: X \rightarrow 2^{Y}$ be l.s.c., $A \subset X$ closed, and $g$ a selection for $\dot{\phi} \mid A$. Define $\dot{\phi}_{g}: X \rightarrow 2^{Y}$ by $\dot{\phi}_{g}(x)=\dot{\phi}(x)$ if $x \in X-A$ and $\phi_{g}(x)=\{g(x)\}$ if $x \in A$. Then $\phi_{g}$ is also l.s.c.

Henceforth, we shall freely refer to the map $\phi_{g}$ defined in Lemma 2.1.

Lemma 2.2 [4, Lemma 11.3]. Let $\phi: X \rightarrow 2^{Y}$ be 1.s.c. with $Y$ a metric space, let $K \subset Y$ be compact, and let $\varepsilon>0$. Then $\{x \in X$ : $\left.K \subset B_{i}(\phi(x))\right\}^{3}$ is open in $Y$.

3. Two properties of set-valued maps. In this section we consider the following two properties of a map $\phi: X \rightarrow 2^{Y}$, where $Y$ is a metric space.

(3.1) To every $\varepsilon>0$ corresponds an $\alpha(\varepsilon)>0$ with the following property: If $A \subset X$ is closed, $g$ is a selection for $\phi \mid A$, and $h: X \rightarrow Y$ is continuous with $d\left(h, \dot{\phi}_{g}\right)<\alpha(\varepsilon)$, then $g$ extends to a selection $f$ for $\phi$ with $d(f, h)<\varepsilon{ }^{4}$

(3.2) To every $\varepsilon>0$ corresponds a $\beta(\varepsilon)>0$ with the following property: If $A \subset X$ is closed, $g$ is a selection for $\phi \mid A, h: X \rightarrow Y$ is continuous with $d\left(h, \dot{\phi}_{g}\right)<\beta(\varepsilon)$, and $\mu>0$, then there exists a continuous $f: X \rightarrow Y$ with $d(f, h)<\varepsilon$ and $d\left(f, \dot{\phi}_{g}\right)<\mu$.

Property (3.2) (often with $A=\varnothing$ ) is used-implicitly or explicitly -in the proofs of almost all general selection theorems known to the author, including those in this paper. It is clear that the slightly simpler property (3.1) (which is called the selection $a p$ proximation property, or SAP, in $[6, \S 5]$ ) always implies (3.2); the

${ }^{2}$ i.e., $\phi$ satisfies the definition of the SNEP for this particular $A$.

${ }_{3} B_{\varepsilon}(S)$ denotes the $\varepsilon$-neighborhood of $S$.

${ }_{4}^{4}$ Here $d$ is the metric on $Y$, and $d\left(h, \phi_{g}\right)<\alpha$ denotes that $d\left(h(x), \phi_{g}(x)\right)<\alpha$ for all $x \in X$. Similarly for $d(f, h)<\varepsilon$, etc. 
following result shows that, under mild restrictions, the two properties are actually equivalent.

Proposition 3.3. If $Y$ is a complete metric space, and if $\phi(x)$ is closed in $Y$ for all $x \in X$, then (3.2) implies (3.1). Moreover, one can take $\alpha(\varepsilon)=\beta(\varepsilon / 2)$; in particular, if one can take $\beta(\infty)=\infty^{5}$ then one can also take $\alpha(\infty)=\infty$.

Proof. Assume that $\phi$ satisfies (3.2) and let us show that it satisfies (3.1) with $\alpha(\varepsilon)=\beta(\varepsilon / 2)$. Let $f_{1}=h$. By induction, use (3.2) to construct continuous maps $f_{n}: X \rightarrow Y$ such that $d\left(f_{n}, \phi_{g}\right)<\beta\left(2^{-n} \varepsilon\right)$ and $d\left(f_{n+1}, f_{n}\right)<2^{-(n+1)} \varepsilon$ for all $n \geqq 1$. Let $f=\lim _{n} f_{n}$. This $f$ satisfies (3.1) with $\alpha(\varepsilon)=\beta(\varepsilon / 2)$.

Proposition 3.4. Suppose $\phi: X \rightarrow 2^{Y}$, with $Y$ metric. Then:

(a) If $\phi$ satisfies (3.1) with $\alpha(\infty)=\infty$, then $\phi$ has the SEP.

(b) If $X$ is collectionwise normal ${ }^{6}$, if $Y$ is a Banach space, and if $\phi \mid B$ satisfies (3.1) for every closed $B \subset X$ with $\alpha(\infty)$ independent of $B$, then $\phi$ has the SNEP.

Proof. (a) Clear (see Footnote 5).

(b) Suppose $A \subset X$ is closed and $g$ is a selection for $\phi \mid A$. By a theorem of C. H. Dowker [1], $g$ can be extended to a continuous $h: X \rightarrow Y$. Let $U=\{x \in X: d(h, \phi)<\alpha(\infty)\}$. Then $U$ is open in $X$ (since $\phi$ is l.s.c.) and $A \subset U$, so there is an open $V$ in $X$ such that $A \subset V \subset \bar{V} \subset U$. Then $d\left(h\left|\bar{V}, \phi_{g}\right| \bar{V}\right)<\alpha(\infty)$, so by assumption $g$ extends to a selection $f$ for $\phi \mid \bar{V}$. That completes the proof.

4. A lemma about nerves of coverings. If $\mathscr{U}$ is a covering of $X$, then we write $N(\mathscr{C})$ for the nerve of $\mathscr{C}$ and $N^{i}(\mathscr{C})$ for the $i$-skeleton of $N(\mathscr{U})$. A function $u: N^{i}(\mathscr{C}) \rightarrow Y$ is called a representation if $u \mid \sigma$ is continuous for every simplex $\sigma$ of $N^{i}(\mathscr{C})$.

LeMma 4.1. Let $Y$ be a Banach space and $\mathscr{S} \subset 2^{Y}$ uniformly equi- $L C^{n}$. Then to every $\varepsilon>0$ corresponds a $\gamma(\varepsilon)>0$ with the following property: If $X$ is paracompact, $\phi: X \rightarrow \mathscr{S}$ l.s.c., $h: X \rightarrow Y$ continuous with $d(h, \phi)<\gamma(\varepsilon)$, and $\mu>0$, then there exists a locally finite open cover $\mathscr{C}$ of $X$ and a representation $u: N^{n+1}(\mathscr{C}) \rightarrow Y$ such that, if $\sigma$ is a simplex of $N^{n+1}(\mathscr{C})$ with vertices $U_{1}, \cdots, U_{j}$, and if $x \in U_{1} \cap \cdots \cap U_{j}$, then

5 That one can take $\alpha(\infty)=\infty$ simply means, in effect, that there exists a selection $f$ for $\phi_{g}$, with nothing of consequence being assumed or concluded about $h$. This useful shorthand, which was introduced in [4], will be used throughout this paper.

${ }_{6}$ This property lies between normality and paracompactniess. 


$$
u(\sigma) \subset B_{\mu}(\phi(x)) \cap B_{\varepsilon}(h(x)) .
$$

If every $S \in \mathscr{S}$ is $C^{n}$, then one can take $\gamma(\infty)=\infty$.

Proof. This result is, in effect, a slight generalization of [4, Lemma 6.1]. In that lemma, it was assumed-in addition to our present hypotheses - that $\operatorname{dim} X \leqq n+1$, and it was concludedin addition to our present conclusions - that the cover $\mathscr{C}$ is of order $\leqq n+2$ (so that $N(\mathscr{C})=N^{n+1}(\mathscr{C})$ ). The proof of [4, Lemma 6.1] remains valid for our present result.

5. Proof of Theorem 1.2. By Propositions 3.3 and 3.4, it will suffice to show that $\phi$ satisfies condition (3.2), ${ }^{7}$ and that one can take $\beta(\infty)=\infty$ in case $\phi(x)$ is $C^{n}$ for every $x \in Z$. In fact, we will show that $\phi$ satisfies $(3.2)$ with $\beta(\varepsilon)=\gamma(\varepsilon)$, where $\gamma(\varepsilon)$ is as in Lemma 4.1.

Let $A, g$ and $h$ be as in (3.2), with $\beta(\varepsilon)=\gamma(\varepsilon)$. Our hypotheses imply that $\left\{\dot{\phi}_{g}(x): x \in X\right\}$ is uniformly equi- $L C^{n}$, so we can pick an open cover $\mathscr{U}$ of $X$ and a representation $u: N^{n+1}(\mathscr{C}) \rightarrow Y$ as in Lemma 4.1 (applied to $\phi_{g}$ ). Now recall that, by Dugundji's extension theorem, every convex subset of a Banach space has the extension property with respect to metric spaces. By inductively climbing up the $i$-skeletons of $N(\mathscr{C})$ with $i>n+1$, we can therefore extend $u$ to a representation $v: N(\mathscr{C}) \rightarrow Y$ such that $v(\sigma) \subset$ $\operatorname{conv} v(\dot{\sigma})$ for every simplex $\sigma$ of $N(\mathscr{C})$ with $\operatorname{dim} \sigma>n+1$. It follows that $v(\sigma) \subset \operatorname{conv} u\left(\sigma \cap N^{n+1}(\mathscr{C})\right)$ for every simplex $\sigma$ of $N(\mathscr{Q})$.

From now on, the proof will closely follow the proof of Theorem 1.1 given in [7].

Let $\left\{V_{U}: U \in \mathscr{Q}\right\}$ be an open cover of $X$ such that $\bar{V}_{U} \subset U$ for all $U \in \mathscr{C}$. For each $x \in X$, let $\sigma(x)$ be the simplex of $N(\mathscr{C})$ spanned by those $U \in \mathscr{C}$ for which $x \in \bar{V}_{U}$. Let $S=X-Z$. For each $s \in S$, let $H_{s}=\bigcup\left\{\bar{V}_{U}: s \notin \bar{V}_{U}\right\}$, and let

$$
G_{s}=\left\{x \in X: v\left(\sigma_{s}\right) \subset\left[B_{u^{\prime}}\left(\dot{\phi}_{g}(x)\right) \cap B_{\varepsilon}(h(x))\right]\right\}-H_{s} .
$$

Then $G_{s}$ is open in $X$ by Lemmas 2.1 and 2.2 , while $s \in G_{s}$ by the properties of $\mathscr{U}$ and $u$ stated in Lemma 4.1, our construction of $v$, and the fact that $B_{\mu}\left(\dot{\phi}_{g}(s)\right)$ and $B_{\varepsilon}(h(s))$ are convex. Also, by the last part of the definition of $G_{s}, \sigma_{x} \subset \sigma_{s}$ for every $x \in G_{s}$.

Let $G=\bigcup_{s \in S} G_{s}$, and let $E=X-G$. Then $E \subset Z$ and $E$ is closed in $X$, so $\operatorname{dim} E \leqq n+1$. Now $\left\{V_{U} \cap E: U \in \mathscr{Q}\right\}$ is a locally finite, relatively open cover of $E$, so it has a relatively open refinement $\left\{O_{l}: U \in \mathscr{L}\right\}$ of (indexed) order $\leqq n+2$ with $O_{U} \subset V_{U}$ for all

${ }^{\top}$ It is worth recording that this will also establish following result: If $\phi$ is as in Theorem 1.2, then $\phi$ has property (3.1). 
$U \in \mathscr{Z}$. Let $W_{U}=V_{U} \cap\left(O_{U} \cup G\right)$. Then $\left\{W_{U}: U \in \mathscr{C}\right\}$ is an open cover of $X, W_{U} \subset V_{U} \subset U$ for all $U \in \mathscr{Q}$, and every $x \in E$ is in $W_{U}$ for at most $n+2$ elements $U \in \mathscr{Q}$. Let $\xi: X \rightarrow N(\mathscr{C})$ be a canonical map obtained by using a partition of unity on $X$ subordinated to $\left\{W_{U}: U \in \mathscr{Q}\right\}$, and define $f: X \rightarrow Y$ by $f=v \circ \xi$. Let us show that $d\left(f, \phi_{g}\right)<\mu$ and $d(f, h)<\varepsilon$, as required by (3.2).

If $x \in E$, then $\xi(x) \in N^{n+1}(\mathscr{C})$, so

$$
f(x)=v(\xi(x))=u(\xi(x)) \in u\left(\sigma_{x}\right) \subset B_{\mu}\left(\phi_{g}(x)\right) \cap B_{\varepsilon}(h(x)),
$$

where the last inclusion holds by our hypotheses on $u$. Now suppose $x \in G$. Then $x \in G_{s}$ for some $s \in S$. Hence $\sigma_{x} \subset \sigma_{s}$, so

$$
f(x)=v(\xi(x)) \in v\left(\sigma_{x}\right) \subset v\left(\sigma_{s}\right) \subset B_{\mu}\left(\phi_{g}(x)\right) \cap B_{\varepsilon}(h(x)),
$$

where the last inclusion holds by the definition of $G_{s}$. That completes the proof.

6. Proof of Theorem 1.3. We begin by recalling a definition from [5]. A metric space $Y$ is a uniform ANR if to every $\varepsilon>0$ corresponds a $\delta(\varepsilon)>0$ with the following property: If $Y$ is embedded isometrically as a closed subset in a metric space $(E, d)$, then there exists a retraction $r: B_{\delta(\infty)}(Y) \rightarrow Y$ such that $d(z, r(z))<\varepsilon$ whenever $z \in E$ and $d(z, Y)<\delta(\varepsilon)$. If one can take $\delta(\infty)=\infty$ (so that the domain of $r$ is always $E$ ), then $Y$ is called a uniform AR.

Before proving Theorem 1.3, we need the following lemma.

Lemma 6.1. Let $\left(Y, d_{0}\right)$ be a metric ANR (resp. AR), and let $\mathscr{S} \subset 2^{Y}$ be equi-LC $C^{n}$ in $Y$. Then there exists a compatible metric $d \geqq d_{0}$ on $Y$ such that:

(a) $(Y, d)$ is a uniform ANR (resp. uniform AR).

(b) $\mathscr{S}$ is uniformly equi- $L C^{n}$ with respect to $d$.

Proof. The existence of a metric $d$ satisfying (a) was proved in $[5 \text {, Theorem 1.1] }]^{8}$, and the existence of a metric $d$ satisfying (b) was proved in [4, Proposition 2.1]. Both results were proved with the aid of [2, Theorem 1], and that theorem can similarly be applied to obtain a metric $d$ satisfying (a) and (b) simultaneously. We omit the details.

Having established Lemma 6.1, we now proceed to the proof of Theorem 1.3. We begin by remetrizing $Y$ with a metric $d$ as in Lemma 6.1, with $\mathscr{S}=\{\phi(x): x \in Z\}$; since the original metric $d_{0}$ on $Y$ complete, so is the metric $d \geqq d_{0}$. Next, we embed $(Y, d)$ isometrically in a Banach space $(E, d)$. Let $\delta(\varepsilon)$ and $r: B_{\delta(\infty)}(Y) \rightarrow Y$ be as in

\footnotetext{
${ }^{8}$ For AR's, it had previously been proved by H. Toruńczyk in [8, Proposition 2.2].
} 
the above definition of a uniform ANR. We shall regard $\phi$ as a map from $X$ to $\mathscr{F}(E)$.

By Propositions 3.3 and 3.4, it will suffice to show that $\phi$ satisfies condition (3.2), ${ }^{9}$ and that one can take $\beta(\infty)=\infty$ in case $Y$ is an $\mathrm{AR}$ and $\phi(x)$ is $C^{n}$ for every $x \in Z$. In fact, we will show that $\phi$ satisfies (3.2) with $\beta(\varepsilon)=\gamma\left(\frac{1}{2} \delta(\varepsilon / 2)\right)$, where $\gamma$ is as in Lemma 4.1 and $\delta$ is as in the above definition of a uniform ANR.

We proceed as in the proof of Theorem 1.2, with a few modifications. Let $A, g$ and $h$ be as in (3.2), with $\beta(\varepsilon)=\gamma\left(\frac{1}{2} \delta(\varepsilon / 2)\right)$. Our hypotheses imply that $\left\{\phi_{g}(x): x \in X\right\}$ is uniformly equi- $L C^{n}$, so we can choose an open cover $\mathscr{C}$ of $X$ and a representation $u: N^{n+1}(\mathscr{U}) \rightarrow E$ as in Lemma 4.1 (applied to $\phi_{g}$ ), but with $\varepsilon$ replaced by $\varepsilon^{\prime}=\frac{1}{2} \delta(\varepsilon / 2)$ and with $\mu$ replaced by $\mu^{\prime}=\delta(\mu / 2)$. In terms of this $\varepsilon^{\prime}$, the above definition of $\beta(\varepsilon)$ becomes $\beta(\varepsilon)=\gamma\left(\varepsilon^{\prime}\right)$, so that $d\left(h, \phi_{g}\right)<\gamma\left(\varepsilon^{\prime}\right)$. Moreover, our choice of $\varepsilon^{\prime}$ and $\mu^{\prime}$ yields the following two assertions, whose easy verification is left to the reader. (Recall that $r: B_{\delta(\infty)}(Y) \rightarrow Y$ is our retraction.)

(1) If $x \in X, e \in E$, and $e \in B_{\varepsilon^{\prime}}(h(x))$, then $e \in B_{\delta(\varepsilon / 2)}(Y)$ and $r(e) \in B_{\varepsilon}(h(x))$.

(2) If $x \in A, e \in E$ and $e \in B_{\mu^{\prime}}(g(x))$, then $r(e) \in B_{\mu}(g(x))$.

Now extend the representation $u: N^{n+1}(\mathscr{C}) \rightarrow E$ to a representation $v: N(\mathscr{U}) \rightarrow E$ precisely as in the proof of Theorem 1.2. The properties of $\mathscr{U}, u$ and $v$ imply that, if $\sigma$ is a simplex of $N(\mathscr{C})$ and if $x$ is in the intersection of its vertices, then $v(\sigma) \subset B_{\varepsilon^{\prime}}(h(x))$, so $v(\sigma) \subset B_{\delta(\infty)}(Y)$ by (1). It follows that $v(N(\mathscr{C}))$ is contained in the domain of $r$, and we can define $w=r \circ v$. Our proof now continues just like the proof of Theorem 1.2, except that $v$ is replaced by $w$ in the definitions of $G_{s}$ and $f$. Everything goes through as before, except that it requires a bit more care to check that $s \in G_{s}$ and that $f(x) \in B_{\varepsilon}(h(x)) \cap B_{\mu}\left(\phi_{g}(x)\right)$ when $x \in E$.

(a) $s \in G_{s}$ : We must check that $w\left(\sigma_{s}\right) \subset B_{\varepsilon}(h(s))$ and that $w\left(\sigma_{s}\right) \subset$ $B_{\mu}\left(\phi_{g}(s)\right)$. For the first inclusion, observe that $v\left(\sigma_{s}\right) \subset B_{\varepsilon^{\prime}}(h(s))$ by the previous paragraph, so $w\left(\sigma_{s}\right) \subset B_{\varepsilon}(h(s))$ by (1). For the second inclusion, we distinguish two cases: If $s \notin A$, then $\phi_{g}(s)=Y$, so our inclusion is clear because $w\left(\sigma_{s}\right) \subset Y$. If $s \in A$, then $\phi_{g}(s)=\{g(s)\}$; since $u\left(\sigma_{s}\right) \subset B_{\mu^{\prime}}\left(\phi_{g}(s)\right)$ by Lemma 4.1 , we have $u\left(\sigma_{s}\right) \subset B_{\mu^{\prime}}(g(s))$, hence $v\left(\sigma_{s}\right) \subset B_{\mu}(g(s))$ by the construction of $v$, so (2) implies that $w\left(\sigma_{s}\right) \subset$ $B_{\mu}(g(s))=B_{\mu}\left(\phi_{g}(s)\right)$.

(b) $f(x) \in B_{\varepsilon}(h(x)) \cap B_{\mu}\left(\phi_{g}(x)\right)$ when $x \in E$ : Just as in the proof of Theorem 1.2, we have $v(\xi(x)) \in B_{\varepsilon^{\prime}}(h(x)) \cap B_{\mu^{\prime}}\left(\dot{\phi}_{g}(x)\right)$ whenever $x \in E$; since $f(x)=r(v(\xi(x)))$, our assertion follows from (1) and (2).

That completes the proof.

${ }^{9}$ It is worth recording that this will also establish the following result: If $\phi$ : $X \rightarrow \mathscr{F}(Y)$ is as in Theorem 1.3, then $Y$ can be remetrized so that $\phi$ has property (3.1). 
REMARK. Since the above proof of Theorem 1.3 is fairly complicated, it may be worthwhile to outline an alternative proof for the important special case where $n=-1$ : First of all, we now only need part (a) of Lemma 6.1. Next, let us show that (3.2) is satisfied with $\beta(\varepsilon)=\frac{1}{2} \delta(\varepsilon / 2)$, where $\delta$ is as in the definition of a uniform ANR: With $h$ and $\phi_{g}$ as in (3.2), define

$$
\begin{aligned}
\theta(x) & =\phi_{g}(x) \cap B_{\beta(\varepsilon)}(h(x)), \\
\psi(x) & =\left[\operatorname{conv}(\theta(x)) \cap r^{-1}\left(B_{\mu / 2}(\phi(x))\right)\right]^{-} .
\end{aligned}
$$

Standard facts about l.s.c. maps imply that $\psi$ is l.s.c. Now $\psi(x)=$ [conv $(\theta(x))]^{-}$for all $x \in Z$, so $\psi$ has a selection $f^{\prime}$ by Theorem $1.1 .^{10}$ It is easy to check that $f^{\prime}(x)$ is in $B_{\delta(\varepsilon / 2)}(Y)$ - and thus in the domain of $r$-for all $x \in X$, so we can define $f=r \circ f^{\prime}$. It is not hard to check that this $f$ is an extension of $g$ which satisfies all the requirements of (3.2).

7. Proof of Theorem 1.4. As observed in the introduction, this theorem reduces to [4, Theorem 1.2] when $Z=\varnothing$. The proof of Theorem 1.4 is very similar to that of the older result given in [4], so it will suffice to indicate the necessary modifications.

(1) Analogously to [4, Proposition 2.1], one proves that there exists a compatible complete metric $d$ on $Y$ which simultaneously makes $\{\phi(x): x \in X-Z\}$ uniformly $L C^{n}$ and $\{\phi(x): x \in Z\}$ uniformly equi- $L C^{m}$. (Observe that $\{\phi(x): x \in X\}$ is therefore also uniformly equi- $L C^{m}$.)

(2) After the modification in (1) above, we can follow the proof of [4, Theorem 1.2] essentially without change until we get to the proof of [4, Lemma 7.3]. In that proof, the hypothesis that $\{\phi(x): x \in X\}$ is uniformly equi- $L C^{n}$ was needed to construct the maps $u_{\sigma, x}$ (for $\operatorname{dim} \sigma=i+1$ ) in the middle of p. 572 of [4]. Since $\{\phi(x)$ : $x \in X\}$ is uniformly equi- $L C^{m}$ in our present situation, we can construct $u_{\sigma, x}$ as before when $i \leqq m$. For $i>m$, however, $u_{\sigma, x}$ can be defined as before only if $x \in X-Z$, but not, in general, when $x \in Z$. To overcome this obstacle, it suffices to modify the proof of $[4$, Lemma 7.3] when $i>m$ by first of all shrinking the given open cover $\mathscr{U}$ of $X$ to an open cover $\mathscr{U}^{\prime}=\left\{U^{\prime}: U \in \mathscr{C}\right\}$ with the following two properties:

(a) $U^{\prime} \subset U$ for all $U \in \mathscr{W}$.

(b) For the cover $\mathscr{U}^{\prime}$, the construction of suitable maps $u_{\sigma, x}^{\prime}$ is possible for all $x \in X$. More precisely: If $\sigma$ is a simplex of

10 This is where we need the assumption that $n=-1$. For $n>-1$, there is no reason to suppose that $\psi$ satisfies the hypotheses of Theorem 1.2. That explains why, in our proof of the general case of Theorem 1.3, we were unable to apply Theorem 1.2 and therefore had to imitate its proof instead. 
$N^{i+1}(\mathscr{Q})$ with $\operatorname{dim} \sigma=i+1$ and with vertices $U_{1}, \cdots, U_{i+2}$, and if $x \in U_{1}^{\prime} \cap \cdots \cap U_{i+2}^{\prime}$ then $u \mid \dot{\sigma}$ can be extended to a continuous $u_{x, \sigma}^{\prime}$ : $\sigma \rightarrow Y$ such that $u_{x, \sigma}^{\prime}(\sigma) \subset B_{\mu}(\phi(x))$ and $\operatorname{diam} u_{x, \sigma}^{\prime}(\sigma)<\varepsilon / 2$.

We construct $\mathscr{U}^{\prime}$ as follows. Let $S=X-Z$. If $\sigma$ is a simplex of $N^{i+1}(\mathscr{U})$ with $\operatorname{dim} \sigma=i+1$, and if $x \in S$ is in the intersection of the vertices of $\sigma$, then (since $x \notin Z$ ) we can define $u_{x, a}, W_{x, \sigma}$ and $W_{x}$ precisely as in the middle of p. 572 of [4]. Now pick an open cover $\left\{U^{*}: U \in \mathscr{C}\right\}$ of $X$ such that $\bar{U}^{*} \subset U$ for all $U \in \mathscr{U}$, and for $s \in S$ let

$$
G_{s}=W_{s}-\bigcup\left\{\bar{U}^{*}: U \in \mathscr{Q}, s \notin \bar{U}^{*}\right\} .
$$

Clearly $G_{s}$ is open in $X$ and $s \in G_{s}$. Let $G=\bigcup_{s \in S} G_{s}$, and let $E=$ $X-G$. Then $E \subset Z$ and $E$ is closed in $X$, so $\operatorname{dim} E \leqq m+1$. Hence the relatively open cover $\left\{U^{*} \cap E: U \in \mathscr{Q}\right\}$ of $E$ has a relatively open refinement $\left\{O_{U}: U \in \mathscr{Q}\right\}$ of (indexed) order $\leqq m+2$ with $O_{U} \subset U^{*}$ for all $U \in \mathscr{Q}$. Now let $U^{\prime}=U^{*} \cap\left(O_{U} \cup G\right)$ for all $U \in \mathscr{Z}$, and let $\mathscr{U}^{\prime}=\left\{U^{\prime}: U \in \mathscr{C}\right\}$. Clearly $\mathscr{U}^{\prime}$ is an open cover of $X$, so let us verify that it satisfies conditions (a) and (b).

That (a) is satisfied is clear, so it remains to check (b). Now if $x \in E$, then (b) is trivially satisfied, since then $x \in U^{\prime}$ for at most $m+2 \leqq i+1$ sets $U \in \mathscr{Z}$. So suppose $x \in G$. Then we can choose $s \in S$ such that $x \in G_{s}$. Now if $\sigma$ and $U_{1}, \cdots, U_{i+2}$ are as in (b), then $x \in U_{1}^{*} \cap \cdots \cap U_{i+2}^{*}$, so the definition of $G_{s}$ implies that

$$
s \in\left(\bar{U}_{1}^{*} \cap \cdots \cap \bar{U}_{i+2}^{*}\right) \subset\left(U_{1} \cap \cdots \cap U_{i+2}\right) .
$$

Thus $s$ is in the intersection of the vertices of $\sigma$, so $u_{s, \sigma}$ and $W_{s, \sigma}$ are defined and $W_{s} \subset W_{s, \sigma}$. Hence $x \in G \subset W_{s} \subset W_{s, \sigma}$. We now let $u_{x, \sigma}^{\prime}=u_{s, \sigma}$. This choice of $u_{x, \sigma}^{\prime}$ satisfies (b) because $x \in W_{s, \sigma}$. That completes the proof.

\section{REFERENCES}

1. C. H. Dowker, On a theorem of Hanner, Ark. Mat., 2 (1952), 307-313.

2. J. Dugundji and E. Michael, On local and uniformly local topological properties, Proc. Amer. Math. Soc., 7 (1956), 304-307.

3. E. Michael, Continuous selections I, Ann. of Math., 63 (1956), 361-382.

4. - Continuous selections II, Ann. of Math., 64 (1956), 562-580.

5. —uniform AR's and ANR's, Compositio Math., 39 (1979), 129-149.

6. Continuous selections and countable sets, to appear in Fund. Math.

7. E. Michael and C. Pixley, A unified theorem on continuous selections, Pacific J. Math., 87 (1980), 187-188.

8. H. Torunczyk, Absolute retracts as factors of normed linear spaces, Fund. Math., 86 (1974), 53-76.

Received May 21, 1979. Supported in part by a grant from the National Science Foundation at the University of Washington and in part by a grant from the Alexander von Humboldt Foundation at the University of Stuttgart.

UNIVERSITY OF WASHINGTON

SeattLe, WA 98195 



\section{PACIFIC JOURNAL OF MATHEMATICS}

\section{EDITORS}

DONALD BABBITT (Managing Editor)

University of Galifornia

Los Angeles, California 90024

HUGo RossI

University of Utah

Salt Lake City, UT 84112

C. C. MOORE AND ANDREW OGG

University of California

Berkeley, CA 94720

\section{J. DUGUNDJI}

Department of Mathematics University of Southern California Los Angeles, California 90007

R. FINN AND J. MILGRAM Stanford University Stanford, California 94305

ASSOCIATE EDITORS
E. F. BECKENBACH
B. H. NeumanN
F. WoLF
K. YosHIDA

\section{SUPPORTING INSTITUTIONS}

UNIVERSITY OF BRITISH COLUMBIA UNIVERSITY OF SOUTHERN CALIFONIA CALIFORNIA INSTITUTE OF TECHNOLOGY UNIVERSITY OF CALIFORNIA MONTANA STATE UNIVERSITY STANFORD UNIVERSITY UNIVERSITY OF HAWAII UNIVERSITY OF TOKYO UNIVERSITY OF NEVADA, RENO UNIVERSITY OF UTAH NEW MEXICO STATE UNIVERSITY WASHINGTON STATE UNIVERSITY OREGON STATE UNIVERSITY UNIVERSITY OF OREGON UNIVERSITY OF WASHINGTON 


\section{Pacific Journal of Mathematics \\ Vol. 87, No. $1 \quad$ January, 1980}

Spiros Argyros, A decomposition of complete Boolean algebras ..........

Gerald A. Beer, The approximation of upper semicontinuous multifunctions

by step multifunctions . . . . ....................

Ehrhard Behrends and Richard Evans, Multiplicity theory for Boolean

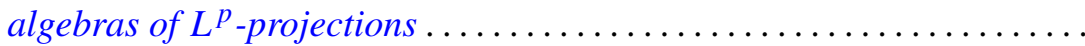

Man-Duen Choi, The full $C^{*}$-algebra of the free group on two

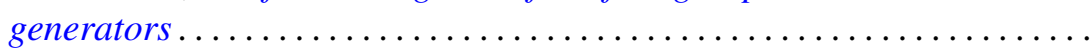

Jen-Chung Chuan, Axioms for closed left ideals in a $C^{*}$-algebra . . . . . . . .

Jo-Ann Deborah Cohen, The strong approximation theorem and locally

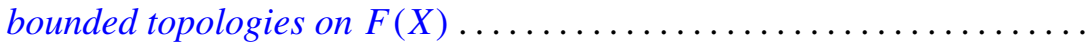

Eugene Harrison Gover and Mark Bernard Ramras, Increasing sequences of Betti numbers............................

Morton Edward Harris, Finite groups having an involution centralizer with

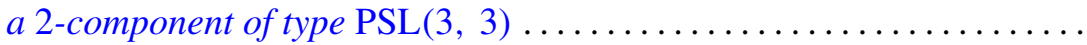

Valéria Botelho de Magalhães Iório, Hopf $C^{*}$-algebras and locally compact

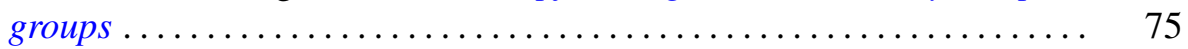

Roy Andrew Johnson, Nearly Borel sets and product measures . . . . . . . . . .

Lowell Edwin Jones, Construction of $Z_{p}$-actions on manifolds . . . . . . . . .

Manuel Lerman and Robert Irving Soare, $d$-simple sets, small sets, and

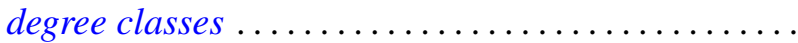

Philip W. McCartney, Neighborly bushes and the Radon-Nikodým property

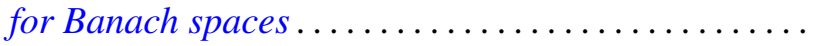

Robert Colman McOwen, Fredholm theory of partial differential equations on complete Riemannian manifolds.

Ernest A. Michael and Carl Preston Pixley, A unified theorem on continuous selections.

Ernest A. Michael, Continuous selections and finite-dimensional sets .

Vassili Nestoridis, Inner functions: noninvariant connected components...

Bun Wong, A maximum principle on Clifford torus and nonexistence of proper holomorphic map from the ball to polydisc.

Steve Wright, Similarity orbits of approximately finite $C^{*}$-algebras . . .

Kenjiro Yanagi, On some fixed point theorems for multivalued

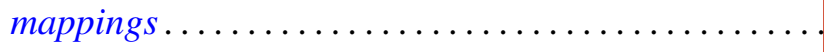

Wieslaw Zelazko, A characterization of LC-nonremovable ideals in commutative Banach algebras 